\title{
Prácticas de alto rendimiento y su incidencia en el compromiso organizacional. Un estudio en colaboradores de una caja de compensación familiar en Colombia
}

DOI: https://doi.org/10.21158/01208160.n90.2021.2976

Fecha de recepción: 18 de junio de 2020

Fecha de aprobación: 26 de mayo de 2021

Fecha de publicación: 05 de agosto de 2021

\author{
Carolina Monsalve-Castro ${ }^{1}$ \\ Universidad Santo Tomás - Bucaramanga \\ caromonsalve83@hotmail.com \\ Fabio Nelson Riaño ${ }^{2}$ \\ Universidad Santo Tomás - Bucaramanga \\ nelsonfaneri@gmail.com \\ Marianela Luzardo-Briceño ${ }^{3}$ \\ Universidad de Medellín- Medellín \\ manelubri@gmail.com
}

Cómo citar este artículo / To reference this article / Comment citer cet article / Para citar este artigo:

Monsalve-Castro, C.; Riaño, F. N.; Luzardo-Briceño, M. (2021). Prácticas de alto rendimiento y su incidencia en el compromiso organizacional. Un estudio en colaboradores de una caja de compensación familiar en Colombia. Revista Escuela de Administración de Negocios, (90), 235-254. DOI: https://doi.org/10.21158/01208160.n90.2021.2976

\section{Resumen}

El artículo tiene como propósito determinar la incidencia de las prácticas de alto rendimiento en el compromiso organizacional de los colaboradores de una caja de compensación familiar ubicada en Colombia. Se realizó una conceptualización de las variables de estudio en la que se apreció la multidimensionalidad del compromiso organizacional y las diferentes clasificaciones de las prácticas de alto rendimiento en mayor medida referenciadas en la literatura. La investigación fue de tipo cuantitativo, correlacional y no experimental de corte transversal. Se utilizó la encuesta como instrumento de recolección, aplicado a una muestra no probabilística por conveniencia de 111 empleados. El tratamiento de la información se realizó a través de un análisis factorial exploratorio y, posteriormente, se llevó a cabo un análisis de regresión logística. El instrumento utilizado para la recolección de datos estuvo compuesto por dos escalas de medición; la primera, relacionada con las prácticas de alto rendimiento y la segunda que representa la variable de compromiso organizacional teniendo en cuenta tres dimensiones: normativa, afectiva y continuidad. Los resultados del estudio demuestran que existe una incidencia positiva de las prácticas de alto rendimiento en la dimensión afectiva y normativa del compromiso, aunque no se logró comprobar la relación con el compromiso de continuidad.

1 Doctora en Dirección de Empresas - Universidad de Valencia, España. Magíster en Administración - Universidad Santo Tomás, Colombia. Administradora de empresas - Universidad Pontificia Bolivariana, Colombia. ORCID: https://orcid.org/0000-0002$1572-8876$

2 Administrador de Empresas - Fundación Universitaria los Libertadores, Colombia. Magister en Administración - Universidad Santo Tomás, Colombia. ORCID: https://orcid.org/0000-0002-3791-8398

3 Doctora en Estadística, Magister Scientiae en Estadística Aplicada. Licenciada en Estadística de la Universidad de Los Andes (ULA), Mérida-Venezuela. ORCID: https://orcid.org/0000-0002-8364-5378 
Palabras clave: prácticas de alto rendimiento; compromiso organizacional; compromiso afectivo; compromiso normativo; compromiso de continuidad; recursos humanos; cajas de compensación familiar.

\title{
High-performance practices and their impact on organizational commitment. A study performed on the employees of a family compensation fund in Colombia
}

\begin{abstract}
The purpose of this article is to determine the incidence of high-performance practices on the organizational commitment of employees in a family compensation fund located in Colombia. We carried out a conceptualization of the study variables in which we could admire the multidimensionality of organizational commitment and the different classifications of high-performance practices referenced in the literature. The research was of a quantitative, correlational, and non-experimental cross-sectional type. The collection instrument was a survey, applied to a non-probabilistic convenience sample of 111 employees. The information was processed by means of an exploratory factor analysis, to subsequently carry out a logistic regression analysis. The instrument used for data collection was composed of two measurement scales: the first one related to high-performance practices, and the second one representing the organizational commitment variable, taking into account three dimensions: normative, affective, and continuity. The results of the study show that there is a positive incidence of high-performance practices in the affective and normative dimension of commitment, although the relationship with the commitment to continuity was not verified.
\end{abstract}

Keywords: high performance practices; organizational commitment; affective commitment; normative commitment; continuity commitment; human resources; family compensation funds.

\section{$\rightarrow$ Práticas de alto desempenho e seu impacto no comprometimento organizacional. Um estudo sobre funcionários de um fundo de compensação familiar na Colômbia}

\section{Resumo}

O objetivo do artigo é determinar a incidência de práticas de alto desempenho no comprometimento organizacional dos funcionários de um fundo de compensação familiar localizado na Colômbia. Realizou-se uma conceituação das variáveis de estudo em que se valorizou a multidimensionalidade do comprometimento organizacional e as diferentes classificações de práticas de alto desempenho mais referenciadas na literatura. A pesquisa foi quantitativa, de correlação e não experimental e transversal. Usou-se um questionário como instrumento de coleta, aplicado a uma amostra não probabilística por conveniência de 111 funcionários. $\mathrm{O}$ tratamento das informações foi realizado por meio de uma análise fatorial exploratória e, posteriormente, foi realizada uma análise de regressão logística. O instrumento utilizado para a coleta de dados consistiu em duas escalas de medida: a primeira, relacionada às práticas de alto desempenho, e a segunda, representando a variável de comprometimento organizacional, levando em consideração três dimensões: normativa, afetiva e continuidade. Os resultados do estudo mostram que existe uma incidência positiva de práticas de alto desempenho na dimensão afetiva e normativa do compromisso, embora não se pôde comprovar a relação com o compromisso de continuidade. 
Palavras-chave: práticas de alto desempenho; compromisso organizacional; compromisso afetivo; compromisso normativo; compromisso de continuidade; recursos humanos; fundos de compensação familiar.

\section{La pratique du haut niveau et ses impacts sur l'engagement au sein de l'entreprise: $\rightarrow$ étude portant sur les employés d'une caisse d'allocation familiale colombienne}

\section{Résumé}

Cet article tentera de déterminer l'incidence de la pratique du haut niveau sur l'engagement des employés d'une caisse d'allocation familiale colombienne. Une conceptualisation des variables de l'étude a été réalisée lors de laquelle la multi dimensionnalité de l'engagement organisationnel et les différentes classifications des pratiques de haut niveau référencées ont été analysées dans une plus large mesure. Il s'agit d'une étude quantitative, corrélationnelle, non expérimentale et transversale servant d'instrument de collecte et appliqué à un échantillon de convenance non probabiliste de 111 employés. Le traitement de l'information a été effectué au travers d'une analyse factorielle exploratoire suivi d'une analyse de régression logistique. Linstrument utilisé pour la collecte des données se compose de deux échelles de mesure; le premier, lié aux pratiques performantes, le second, représentant la variable d'engagement organisationnel, prenant en compte trois dimensions : normative, affective et de continuité. Les résultats de l'étude montrent qu'il existe une incidence positive des pratiques du haut niveau sur les dimensions affectives et normatives de l'engagement organisationnel même si cette relation n’a pas été prouvée dans la continuité.

Mots-clés: pratiques du haut niveau; engagement organisationnel; engagement affectif; engagement normatif; engagement sur la continuité; ressources humaines; fonds d'allocation familiale. 


\section{Introducción}

$\mathrm{L}$ as cajas de compensación familiar en Colombia nacen con la Ley 21 de 1982 con el objetivo de otorgar subsidios familiares a los trabajadores de menores ingresos en el país. Estas instituciones se conciben como organizaciones sin ánimo de lucro que tienen como objetivo liderar la formulación y ejecución de programas en busca de minimizar los problemas sociales y económicos que tiene la población. Su función principal es proporcionar bienestar, además de mejorar la calidad de vida de sus afiliados y sus familias, a través de los diferentes programas asistenciales (Rodríguez-Arévalo, 2012).

Estas instituciones, al igual que las organizaciones con ánimo de lucro, deben enfrentar situaciones administrativas relacionadas con generar ingresos económicos, mejorar la infraestructura para los servicios ofrecidos, la ampliación o diversificación de sus servicios sociales, desarrollar planeación administrativa, incorporar herramientas tecnológicas y vincular recurso humano especializado (Rodríguez, 2012).

Conscientes de la importancia del ser humano para alcanzar los propósitos misionales de las organizaciones, Calderón-Hernández, NaranjoValencia y Álvarez-Giraldo (2010) argumentan cómo el reto que deben asumir las empresas es no concebir el departamento de recursos humanos tan solo como un centro de costo, sino, por el contrario, como un centro de inversión que puede generar ventaja competitiva desde su equipo de trabajo. A esto se le suma lo mencionado por Fragoso, Chambel y Castanheira (2019), para quienes el desafío en la gestión estratégica del recurso humano está encaminado a proponer políticas y prácticas que maximicen el compromiso de los individuos hacia la organización, reconocido como un factor significativo que determina el comportamiento de la fuerza laboral en la compañía (Kloutsiniotis y Mihail, 2017; Yahaya y Ebrahim, 2016).
Singh y Gupta (2015) sostienen que fomentar compromiso organizacional en los colaboradores se considera la principal preocupación de las compañías a la hora de retener individuos talentosos en una economía impulsada por el conocimiento. Esto podría explicarse en palabras de Mowday (1998), quien argumenta que el compromiso en el trabajo puede potencialmente añadir sentido a la vida del individuo, de modo que se genera un incremento en el desempeño de la organización y se reducen los índices de rotación y ausentismo.

Investigaciones anteriores han puesto de manifiesto que para lograr compromiso en los colaboradores se debe implementar las prácticas de alto rendimiento (PAR) en la organización (Arthur, 1994; Delaney y Huselid,1996; Macky y Boxall, 2007; Kloutsiniotis y Mihail, 2017; Takeuchi, Way y Wei Tian, 2018). Por ejemplo, Huselid (1995) sostiene que estas prácticas influencian las habilidades de los empleados a través del desarrollo del capital humano de la empresa. Kehoe y Wright (2013) manifiestan que existe evidencia para afirmar que las PAR funcionan de manera inmediata en el compromiso organizacional, debido a que los individuos perciben un ambiente de apoyo e inversión por parte la compañía. Por su parte, Takeuchi et al. (2018) sostienen que su uso mejora las percepciones de los miembros de la organización sobre su entorno, además de promover el compromiso que implica retribuir con esfuerzo al cumplimiento de las metas de la compañía por tratarlos bien.

No obstante, en Latinoamérica el problema radica en que son escasos los estudios que presenten hallazgos para la comprensión de la relación entre compromiso organizacional y las PAR (Cohen, 2008). Andreassi et al., (2014) realizaron un estudio en 48 países en el que concluyen que era necesario analizar si las prácticas de recursos humanos de Europa o los países anglosajones 
podrían llegar a ser utilizadas de forma exitosa en la fuerza laboral de América Latina. En Colombia, Calderón et al. (2010) sostienen que muy poco se ha analizado cómo los departamentos de recursos humanos vinculan de manera estratégica las prácticas de gestión con las actitudes de sus colaboradores.

Al tomar en consideración estos argumentos teóricos, la investigación busca determinar la incidencia que tienen las PAR en el compromiso organizacional de los colaboradores a partir de la realización de un estudio empírico en una caja de compensación familiar ubicada en Colombia. El estudio se desarrolló bajo un enfoque cuantitativo, correlacional, no experimental de tipo transversal y tuvo dos propósitos fundamentales. El primero, aportar conocimientos a la investigación generada en el área de recursos humanos, reconociendo al individuo como creador de valor en la organización (Batt, 2002). El segundo, documentar y apoyar con información la gestión directiva de la caja de compensación, con el propósito de avanzar hacia la obtención de resultados positivos en su desempeño.

El artículo está compuesto en un primer apartado por la fundamentación teórica que abarca la revisión de literatura realizada a las variables de estudio. Luego, en el segundo apartado se presenta la metodología con la que se desarrolló la investigación. Finalmente, en el tercero se exponen los resultados originados de los análisis estadísticos y se concluye con los hallazgos y su implicación tanto en el ámbito académico como empresarial.

\section{Fundamentación teórica}

\subsection{Compromiso organizacional}

$\mathrm{E}^{1}$ compromiso organizacional es una variable administrativa que ha cobrado gran relevancia en el campo investigativo, especialmente porque existen estudios que prueban su efecto en la construcción de relaciones duraderas entre organización-individuo (Cohen, 2008).

Los primeros aportes al concepto datan de la década de los sesenta, cuando bajo una mirada unidimensional Becker (1960) sostuvo que el compromiso organizacional era un vínculo que establece el individuo con la organización a través de la inversión de tiempo y otros aspectos denominados side-bets ${ }^{4}$. A esta misma corriente se le sumaron Porter et al., (1974, p. 604), quienes definieron el compromiso como «la fuerza que identifica a un individuo y su participación en una organización particular»; además, Salancik (1977, p. 62), para quien es «un estado en el que el empleado queda obligado por sus acciones y por medio de estas, a las creencias que sustentan las actividades y su participación en una empresa». En la última década, Klein, Molloy y Brinsfield (2012) generaron un aporte a esta postura mencionando cómo el compromiso es un vínculo psicológico más que una fuerza que une al individuo con la organización.

No obstante, diversos estudios realizados sobre esta variable han contribuido a establecer que el compromiso organizacional es un constructo multidimensionalidad. Por ejemplo, Angle y Perry (1981) presentan el compromiso bajo dos dimensiones: el compromiso valor, concebido como el deber de apoyar los propósitos de la empresa, y el compromiso a permanecer, el cual se relaciona con la intención a continuar como miembro de la organización.

Por su parte, O’Reilly y Chatman (1986) contribuyeron con la afirmación de que el compromiso organizacional era un concepto comprendido por

\footnotetext{
4 Término utilizado por Becker (1960) para hacer alusión al vínculo que el empleado tiene con la organización, representado por las inversiones que realiza en esta.
} 
tres dimensiones: conformidad, definida como la adopción de actitudes para recibir recompensas; identificación, la cual hace alusión al deseo de permanecer en la compañía; e internalización, que corresponde a la coherencia entre los valores del individuo y la empresa.

Luego, aparece la propuesta de Allen y Meyer (1996, p. 252), quienes argumentan que el compromiso es una «relación psicológica entre el empleado y su organización que hace que sea menos probable que el empleado voluntariamente la abandone». De acuerdo con estos autores, el compromiso está dimensionado en afectivo, lo cual corresponde a la identificación, la participación y al vínculo emocional con la organización; de continuidad, se refiere al compromiso basado en los costos que se generan a partir de la renuncia del empleado a la organización; y normativo, con referencia a la obligación que siente el empleado de permanecer con la compañía.

Posteriormente, Mayer y Schoorman (1992) mencionan que el compromiso podría tener dos dimensiones: valor y de continuidad. La primera establece la aceptación de los objetivos y valores de la empresa. La segunda la definen como el deseo de permanecer como miembro de la compañía. Por otra parte, Jaros et al. (1993) realizaron su propuesta bajo tres dimensiones: afectiva, de continuidad y moral. El compromiso afectivo lo definieron como el grado en el que un individuo está ligado a la organización, bien sea por sentimientos de lealtad, de afecto o bien por sentido de pertenencia. El compromiso de continuidad se refiere al grado en el que el empleado experimenta una sensación de estancamiento en su lugar de trabajo debido a los altos costes que implica dejarlo. Y finalmente, el compromiso moral, establecido como el grado en el que la fuerza laboral está ligada a la organización por la interiorización de los objetivos, los valores y la misión.

Con base en la información presentada se diseñó la tabla 1. Su propósito es agrupar las diferentes propuestas y mostrar la evolución en la caracterización del concepto de compromiso organizacional. No obstante, al día de hoy, la propuesta de Allen y Meyer (1996) se considera una de las más representativas (Bentein et al., 2005; Cohen, 2008), debido a que ha traspasado las barreras culturales (Meyer et al., 2012) y goza de gran aceptación en la comunidad científica (Meyer et al., 2002; Mowday, 1998).

Tabla 1. Dimensiones del compromiso organizativo

\begin{tabular}{|c|c|c|}
\hline Enfoque & Autor & Dimensiones \\
\hline \multirow{3}{*}{ Unidimensional } & Becker (1960) & Conductual \\
\hline & Porter et al. (1974) & Afectivo \\
\hline & Klein, Molloy y Brinsfield (2012) & Vínculo psicológico \\
\hline \multirow{4}{*}{ Dos dimensiones } & \multirow{2}{*}{ Angle y Perry (1981) } & Compromiso de valor \\
\hline & & Compromiso a permanecer \\
\hline & \multirow{2}{*}{ Mayer y Schoorman (1992) } & Compromiso de continuidad \\
\hline & & Compromiso de valor \\
\hline \multirow{9}{*}{ Tres dimensiones } & \multirow{3}{*}{ O’Reilly y Chatman (1986) } & Cumplimiento \\
\hline & & Identificación \\
\hline & & Interiorización \\
\hline & \multirow{3}{*}{ Allen y Meyer (1996) } & Compromiso afectivo \\
\hline & & Compromiso normativo \\
\hline & & Compromiso continuidad \\
\hline & \multirow{3}{*}{ Jaros et al. (1993) } & Afectivo \\
\hline & & Continuidad \\
\hline & & Moral \\
\hline
\end{tabular}

Fuente. Elaboración propia 


\subsection{Prácticas de alto rendimiento}

Las prácticas de recursos humanos es un tema que ha despertado gran interés, debido a la importancia que se le ha atribuido a la gestión de personal en el interior de las empresas en los últimos años (Haar, O'Kane y Daellenbach, 2021; Kloutsiniotis, Katou y Mihail, 2021; Sun y Mamman, 2021). De manera inicial, el término de prácticas de alto rendimiento fue incorporado alrededor de los años noventa cuando algunos autores (Arthur, 1994; Huselid, 1995; Delaney y Huselid, 1996) iniciaron las investigaciones sobre la relación entre las PAR y el desempeño de la organización, de modo que notaron relaciones de sinergia entre estas y encontraron evidencia científica de su efecto positivo en el rendimiento.
Para Datta, Guthrie y Wright, las PAR son prácticas de recursos humanos «diseñadas para mejorar las habilidades, el compromiso y la productividad de los empleados» (2005, p. 135) a partir de la creación de condiciones que permitan alcanzar los objetivos de la compañía. Según Para-González, Jiménez-Jiménez y Martínez-Lorente (2019), las PAR podrían mejorar los resultados de los colaboradores mediante la generación de un sentido de pertenencia a la organización como grupo, de manera que se fomente el compromiso afectivo y el empoderamiento de la fuerza laboral. Dados los diferentes conceptos sobre PAR que ofrece la literatura, se presentan en la tabla 2 las principales propuestas identificadas.

Tabla 2. Conceptualización de prácticas de alto rendimiento

\begin{tabular}{|c|l|}
\hline Autor & \multicolumn{1}{c|}{ Concepto } \\
\hline Lado y Wilson (1994) & $\begin{array}{l}\text { Un set de distintas pero relacionadas actividades, funciones y procesos dirigidos a atraer, } \\
\text { desarrollar y mantener al personal de una firma. }\end{array}$ \\
\hline Huselid (1995) & $\begin{array}{l}\text { Prácticas de gestión de recursos humanos que pueden mejorar el conocimiento, las } \\
\text { capacidades y las habilidades de los empleados actuales y potenciales, de manera que se } \\
\text { incremente su motivación, se reduzca su rotación y mejore la retención de empleados de } \\
\text { calidad. }\end{array}$ \\
\hline Guthrie (2001) & $\begin{array}{l}\text { Un sistema de recursos humanos permite una ventaja competitiva sostenible en la medida } \\
\text { en que ayuda a crear una fuerza de trabajo cuyas contribuciones son valiosas, únicas y } \\
\text { difíciles para los competidores de imitar. }\end{array}$ \\
\hline Way (2002) & $\begin{array}{l}\text { Conjunto de prácticas distintas pero interrelacionadas que en conjunto seleccionan, } \\
\text { desarrollan, retienen y motivan a la fuerza de trabajo. }\end{array}$ \\
\hline Ferrer (2014) & $\begin{array}{l}\text { Paquete de herramientas destinadas a la formación y al desarrollo del capital humano; } \\
\text { como segunda instancia, su fin es motivar y fidelizar a los trabajadores. }\end{array}$ \\
\hline Haar et al. (2021) & $\begin{array}{l}\text { Las PAR pueden desarrollar una fuerza laboral superior y continuar mejorando el } \\
\text { conocimiento, las habilidades y las capacidades de los individuos, lo cual permita obtener } \\
\text { un mejor rendimiento con relación al de la competencia. }\end{array}$ \\
\hline
\end{tabular}

Fuente. Elaboración propia.

Por otra parte, en la literatura se logra evidenciar cómo los sistemas de prácticas han sido agrupados por diferentes autores, quienes sostienen que no existe unanimidad en cuanto a cuáles deberían conformar las PAR de una organización (Combs et al., 2006). Por tanto, en la tabla 3 se presentan las diferentes propuestas identificadas en la revisión de literatura. 
Tabla 3. Prácticas de alto rendimiento por diferentes autores

\begin{tabular}{|c|c|}
\hline Autores & Prácticas de recursos humanos \\
\hline Arthur (1994) & $\begin{array}{l}\text { Definición de puestos, participación, resolución de conflictos, } \\
\text { información, trabajadores cualificados, equipos autogestionados, } \\
\text { aprendizaje, beneficios, salarios yacciones. }\end{array}$ \\
\hline Huselid (1995) & $\begin{array}{l}\text { Selección, análisis de rendimiento, compensación, diseño de puestos } \\
\text { de trabajo, quejas, información, evaluación de la actitud, participación, } \\
\text { reclutamiento, promoción y formación. }\end{array}$ \\
\hline McDuffie (1995) & $\begin{array}{l}\text { Equipos autónomos, participación, rotación, descentralización, } \\
\text { reclutamiento, compensación, reducción de estatus, aprendizaje y } \\
\text { formación. }\end{array}$ \\
\hline Delery y Doty (1996) & $\begin{array}{l}\text { Oportunidades de carrera, entrenamiento, orientación a los } \\
\text { resultados, ganancias compartidas, seguridad laboral, participación y } \\
\text { descripción del puesto de trabajo. }\end{array}$ \\
\hline Becker y Huselid (1998) & $\begin{array}{l}\text { Selección, análisis de rendimiento, compensación, diseño de } \\
\text { puestos, quejas, información, evaluación de la actitud, participación, } \\
\text { reclutamiento, formación y promoción. }\end{array}$ \\
\hline Pfeffer (1998) & $\begin{array}{l}\text { Estabilidad, contratación, formación, información, retribución, } \\
\text { reducción de estatus y trabajo en equipo. }\end{array}$ \\
\hline Macky y Boxall (2007) & $\begin{array}{l}\text { Retribución por rendimiento, trabajo en equipo, participación, } \\
\text { diferencias de estatus, promoción, evaluación de rendimiento, } \\
\text { retroalimentación, comunicación, evaluación de la actitud, formación, } \\
\text { selección, quejas y diseño de puestos. }\end{array}$ \\
\hline Chuang y Liao (2010) & $\begin{array}{l}\text { Dotación de personal, capacitación, participación, evaluaciones de } \\
\text { desempeño, compensación/recompensas y bienestar. }\end{array}$ \\
\hline Chuang, Chen y Chuang (2013) & $\begin{array}{l}\text { Prácticas de oportunidad, prácticas de motivación y prácticas de } \\
\text { habilidad. }\end{array}$ \\
\hline Para-González et al. (2019) & Selección, capacitación, evaluación de desempeño y compensación. \\
\hline Miao et al. (2021) & $\begin{array}{l}\text { Capacitación extensa, empoderamiento, evaluación del desempeño } \\
\text { orientada a resultados, gestión de competencias y la disciplina de los } \\
\text { empleados, selección de empleados, intercambio de información y } \\
\text { gestión de recompensas. }\end{array}$ \\
\hline
\end{tabular}

Fuente. Elaboración propia.

Después de observar los diferentes sistemas de prácticas es importante mencionar que el estudio de las PAR se ha realizado bajo la mirada de dos perspectivas teóricas. La primera, denominada «la teoría de recursos y capacidades», en la que se considera a los individuos como activos que pueden convertirse en fuente de ventaja competitiva al ser gestionados por medio de PAR, puesto que los conocimientos y las habilidades que desarrollan contribuyen a que se conviertan en valiosos, raros, difícilmente imitables y apropiables por la organización (Barney, 1991; Wong y Gao, 2014). La segunda, conocida como «enfoque AMO» - por sus siglas en inglés, ability, motivation, opportunity-, sugiere que las PAR conducen a la selección de una fuerza laboral con habilidades superiores, en la cual las oportunidades de capacitación más sólidas darán como resultado una mayor productividad y, de manera similar, las prácticas de compensación apropiadas mejorarán el desempeño a través de una mayor motivación (Haar et al., 2021).

\subsection{Relación entre compromiso organiza- cional y prácticas de alto rendimiento}

Existe un número significativo de publicaciones que abordan la variable de compromiso organizacional vinculada a las prácticas de alto rendimiento 
(Almutawa, Muenjohn y Zhang, 2016; Andersén y Andersén, 2019; Fabi, Lacoursière y Raymond, 2015; Fragoso et al., 2019; Hu et al., 2019; Kehoe y Wright, 2013; Mahmood et al., 2019; Para-González et al., 2019; Pattnaik y Sahoo, 2019; Rhee, Oh y Yu, 2018). Durante años este tipo de prácticas las han adoptado en todo el mundo organizaciones de todos los tamaños (Sun y Mamman, 2021) y este creciente interés se origina en la evidencia de cómo, al utilizar las PAR, los individuos generan esfuerzos superiores provenientes de su compromiso con lograr las metas de la organización (Arthur,1994; Mahmood et al., 2019; Para-González et. al., 2019; Whitener, 2001).

En las investigaciones realizadas sobre el tema se ha podido establecer que el efecto de las PAR en el compromiso de los colaboradores está fundamentado en un concepto de reciprocidad expuesto en la teoría de intercambio social, en el cual tanto el individuo como la organización esperan obtener un beneficio mutuo (Blau,1964). Esto quiere decir que los colaboradores perciben las prácticas como una inversión que la organización otorga para su crecimiento y desarrollo, razón por la que retribuyen con su esfuerzo a fin de mejorar el desempeño de la compañía (Rhee et al., 2018). En otras palabras, si las actividades de gestión son relacionadas con aspectos de equidad, reconocimiento e inversión por empleados, se esperará que respondan con comportamientos deseados, de modo que se genera un efecto positivo en los resultados de la organización (Fragoso et al., 2019; Para-González et al.,2019).

Bajo esta misma mirada, Kehoe y Wright (2013) sostienen que las PAR representan una estrategia invaluable capaz de mejorar el compromiso organizacional, expresado como una consecuencia actitudinal de un tipo de intercambio mutuo desde la perspectiva del individuo. Por ejemplo, Andersén y Andersén (2019) realizaron un estudio en un grupo de colaboradores de medianas y pequeñas empresas de Suecia, en el cual pudieron demostrar que existe una relación positiva entre PAR, género femenino, cargos gerenciales y compromiso afectivo. De acuerdo con los autores, esto podría deberse a dos razones. La primera, a que las organizaciones que implementan PAR pueden ser menos discriminatorias con las mujeres porque se basan en hechos más objetivos al aplicar prácticas relacionadas con el salario o la promoción; la segunda, quienes ocupan cargos de gerencia están directamente involucrados y familiarizados con las prácticas, razón por la cual se identifican fuertemente con ellas.

Otros estudios que vinculan estas dos variables se han realizado en los últimos años. Hu et al. (2019) hallaron cómo los individuos que consideraban tener mayores oportunidades de emplearse por fuera de la organización tenían menos probabilidad de que su compromiso afectivo pudiera verse afectado por la gestión de recursos humanos, al igual que aquellos que llevaban menos tiempo de vinculación. Pattnaik y Sahoo (2019), por su parte, establecieron que las PAR no se relacionaban de manera directa con el desempeño de la organización, pero sí lograron comprobar que el compromiso ayuda a la organización a alcanzar un mejor desempeño cuando utilizan este tipo de prácticas.

Por su parte, Fabi et al. (2015) comprobaron el efecto sinérgico que se le puede atribuir a las PAR bajo la clasificación del enfoque AMO, lo cual podría explicar su efecto en el compromiso organizacional; parafraseando a Almutawa et al. (2016), si los empleados no perciben que dichas prácticas realmente potencian sus habilidades, los motivan adecuadamente y les otorgan la oportunidad de participar, manifestarán poco o ningún compromiso.

Finalmente, esta revisión de literatura permitió destacar el vigente interés por seguir analizando el efecto que pueden generar las PAR en el compromiso de los individuos. Esto con el propósito de aportar tanto a la gestión administrativa como a las posturas emergentes que mencionan cómo cada conjunto de prácticas transmite su propio mensaje 
y, por tanto, es deber de la dirección de recursos humanos velar por la creación de esquemas interpretativos que promuevan la generación de compromiso en los colaboradores con miras a cumplir con los objetivos de la empresa (Mierlo, Bondarouk y Sanders, 2018).

\section{Metodología}

\subsection{Muestra y recolección de información}

耳 ste trabajo de investigación es de tipo - cuantitativo, su alcance es correlacional de corte transversal, no experimental. Parallevar a cabo el empírico se utilizó como unidad de medida a los individuos vinculados a la caja de compensación familiar siguiendo las recomendaciones realizadas por Khilji y Wang (2006), Macky y Boxall, (2007), Nishii, Lepak y Schneider (2008) y Mierlo et al. (2018), quienes sostienen que es necesario continuar indagando sobre cómo se perciben las PAR, no solo desde la mirada de la dirección de talento humano, sino trambién desde la interpretación que los colaboradores le atribuyen en la organización.

Por tal motivo, se decidió realizar de manera inicial un censo poblacional con un total de 129 colaboradores vinculados directamente a la caja de compensación familiar. No obstante, por problemas de orden público en la zona geográfica en la que se ubica la institución, se debió realizar un muestreo no probabilístico por conveniencia en el que participaron 111 colaboradores de fácil acceso con contrato de trabajo directo, lo cual equivale al $86,05 \%$ del total de la población.

Los datos demográficos obtenidos de los participantes permitieron ofrecer una caracterización de la muestra, con la aclaración de que el 51,35 \% corresponde a mujeres y el 48,65 \% a hombres. El 41,44\% corresponde a edades entre los 26 y los 35 años, mientras que el $33,33 \%$ se encuentra en edades entre los
36 y 45 años. También se observó que el 47,75 \% de los individuos son solteros y el $32,43 \%$ se encuentra en unión libre. El nivel educativo de los participantes fue de un $39,64 \%$ profesional y un $13,51 \%$ especialista. Por otro lado, el 38,74 \% de los empleados lleva entre uno y tres años trabajando en la empresa y solo el 14,41 \% de los individuos llevan menos de un año.

La recolección de información se llevó a cabo mediante reuniones grupales en cada una de las sedes y centros de operación de la empresa. Posteriormente, se hizo entrega a cada uno del instrumento y se les otorgó un máximo de 15 minutos para diligenciar el formato. Finalmente, la tabulación y organización de la información obtenida se realizó en Microsoft Office Excel para su posterior análisis en el software SPSS versión 23.

\subsection{Instrumentos de medición}

El instrumento utilizado para la recolección de datos estuvo compuesto por dos escalas de medición. La primera, relacionada con las prácticas de alto rendimiento (véase la Tabla 4), en la cual se utilizó la propuesta realizada por Macky y Boxall (2007), quienes se apoyaron en estudios llevados a cabo por Huselid (1995), Guthrie (2001) y Wright, Gardner y Moynihan (2003); por consiguiente, se acercan a las acciones que realiza el departamento de talento humano de la caja de compensación. La segunda escala representa la variable de compromiso organizacional (véase la Tabla 5), en la cual se tomó la propuesta de Meyer, Allen y Smith (1993), revisada en Meyer y Allen (1997). 
Tabla 4. Indicadores de medición de prácticas de alto rendimiento

\begin{tabular}{|c|c|c|}
\hline Ítems & Prácticas & Indicador de medición \\
\hline 1 & \multirow{2}{*}{$\begin{array}{l}\text { Desempeño basado en el } \\
\text { pago }\end{array}$} & $\begin{array}{l}\text { ¿Ha recibido un pago adicional o un aumento salarial en el pasado año, como } \\
\text { resultado de su desempeño en el trabajo o por trabajar en equipo? }\end{array}$ \\
\hline 2 & & $\begin{array}{l}\text { ¿Tiene su empleador un esquema de participación en las ganancias o de } \\
\text { participación en acciones en el que pueda participar? }\end{array}$ \\
\hline 3 & Trabajo en equipo & $\begin{array}{l}\text { Mi trabajo me obliga a trabajar en estrecha colaboración con otros miembros } \\
\text { del equipo para lograr un objetivo común u objetivo resultado. }\end{array}$ \\
\hline 4 & Participación & $\begin{array}{l}\text { Los empleados aquí tienen la oportunidad de involucrarse en la toma de } \\
\text { decisiones de las cosas que importan. }\end{array}$ \\
\hline 5 & Reducción de estatus & $\begin{array}{l}\text { Hay pocas diferencias de estatus entre los directivos y el resto de los empleados } \\
\text { de mi organización. Todos estamos en el mismo nivel. }\end{array}$ \\
\hline 6 & Promoción interna & $\begin{array}{l}\text { Cuando hay vacantes de trabajo, la gestión normalmente trata de llenarlos } \\
\text { primero con la gente que está dentro de la organización, en lugar de contratar } \\
\text { gente de afuera. }\end{array}$ \\
\hline 7 & \multirow{2}{*}{$\begin{array}{l}\text { Sistemas formales de } \\
\text { evaluación del desempeño }\end{array}$} & $\begin{array}{l}\text { ¿Ha sido su desempeño en el trabajo valorado formalmente por su jefe en los } \\
\text { últimos } 12 \text { meses? }\end{array}$ \\
\hline 8 & & Las evaluaciones de mi trabajo son justas y precisas. \\
\hline 9 & Progreso valorativo & Recibo información periódica y constructiva de lo bien que hago mi trabajo. \\
\hline 10 & $\begin{array}{l}\text { Programas de } \\
\text { comunicación formal }\end{array}$ & $\begin{array}{l}\text { La dirección me mantiene bien informado acerca de la caja de compensación y } \\
\text { de los resultados de su gestión. }\end{array}$ \\
\hline 11 & $\begin{array}{l}\text { Uso regular de encuestas de } \\
\text { actitud de los empleados }\end{array}$ & $\begin{array}{l}\text { ¿Ha participado en una encuesta en la actitud de los empleados llevada a cabo } \\
\text { por la caja de compensación en los últimos dos años? }\end{array}$ \\
\hline 12 & Entrenamiento & Mi empleador me proporciona oportunidades de capacitación y desarrollo. \\
\hline 13 & $\begin{array}{l}\text { Méritos basados en la } \\
\text { promoción }\end{array}$ & $\begin{array}{l}\text { Tengo buenas oportunidades para avanzar en mi carrera a través de un ascenso. } \\
\text { El proceso de promoción utilizado aquí es justo para todos los empleados. }\end{array}$ \\
\hline 14 & Estructura del cargo & $\begin{array}{l}\text { Tengo una descripción del trabajo que detalla con precisión el trabajo que } \\
\text { debo realizar. }\end{array}$ \\
\hline
\end{tabular}

Fuente. Elaboración propia con base en Macky y Boxall, 2007.

Tabla 5. Ítems de compromiso organizacional

\begin{tabular}{|c|c|c|}
\hline Ítems & Dimensión & Indicadores a medir \\
\hline 15 & \multirow{6}{*}{ Normativa } & No siento ninguna obligación de continuar con mi patrón actual. \\
\hline 16 & & Me sentiría culpable si renunciara a mi organización en este momento \\
\hline 17 & & $\begin{array}{l}\text { Incluso si fuera para mi beneficio, no siento que estaría bien dejar ahora mi } \\
\text { organización. }\end{array}$ \\
\hline 18 & & Esta organización merece mi lealtad. \\
\hline 19 & & $\begin{array}{l}\text { No dejaría mi organización ahora mismo porque tengo un sentido de obligación } \\
\text { con las personas de esta. }\end{array}$ \\
\hline 20 & & Le debo mucho a mi organización. \\
\hline 21 & \multirow{6}{*}{ Afectiva } & Estaría muy contento de pasar el resto de mi carrera en esta organización. \\
\hline 22 & & Realmente siento como propios los problemas de esta organización. \\
\hline 23 & & Siento un fuerte sentido de pertenencia a mi organización. \\
\hline 24 & & Me siento emocionalmente unido a esta organización. \\
\hline 25 & & Me siento como «parte de una familia» en mi organización. \\
\hline 26 & & Esta organización tiene un gran significado personal para mí. \\
\hline
\end{tabular}




\begin{tabular}{|c|c|c|}
\hline 27 & \multirow{6}{*}{ Continuidad } & $\begin{array}{l}\text { Ahora mismo, permanecer en mi organización es una cuestión de necesidad más } \\
\text { que deseo. }\end{array}$ \\
\hline 28 & & Aunque quisiera, sería muy duro para mí dejar mi organización ahora. \\
\hline 29 & & $\begin{array}{l}\text { Demasiado en mi vida se vería trastocado si decidiera dejar mi organización ahora } \\
\text { mismo. }\end{array}$ \\
\hline 30 & & $\begin{array}{l}\text { Creo que tengo muy pocas opciones para considerar marcharme de esta } \\
\text { organización. }\end{array}$ \\
\hline 31 & & $\begin{array}{l}\text { Si no hubiese puesto ya tanto de mí mismo en esta organización, podría considerar } \\
\text { trabajar en otro lugar. }\end{array}$ \\
\hline 32 & & $\begin{array}{l}\text { Una de las pocas consecuencias negativas de abandonar esta organización podría } \\
\text { ser la escasez de alternativas disponibles. }\end{array}$ \\
\hline
\end{tabular}

Fuente. Elaboración propia con base en Meyer et al., 1993; Meyer y Allen, 1997.

En total, la encuesta estuvo comprendida por 32 ítems y el tipo de respuesta empleado para ambos casos fue escala Likert, en un rango de 1 a 7 , en las que 1 es completamente en desacuerdo y 7 completamente de acuerdo. En algunas preguntas de las PAR, la respuesta era de carácter dicotómico - p. ej., ¿ha sido su desempeño en el trabajo valorado formalmente por su jefe en los últimos 12 meses? - .

Con respecto a la fiabilidad de la consistencia interna de los ítems que conformaron la escala de medida del instrumento, se utilizó para las dos variables el alfa de Cronbach. Con relación a las PAR se obtuvo un 0,934 , mientras que para el compromiso organizacional el resultado fue de 0,692. Según George y Mallery (2013), los valores que se recomiendan para este indicador deben superar el 0,8 , aunque manifiestan que son válidos aquellos que estén cercanos al 0,7. En este sentido, se asume que los ítems miden el constructo que representan y se correlacionan altamente entre sí (Frías-Navarro, 2020).

\subsection{Análisis de la información}

A fin de establecer la relación entre las variables del estudio se utilizó IBM SPSS versión 23. El análisis estadístico se realizó en dos partes: una primera parte descriptiva y la segunda inferencial. La primera aportó información sociodemográfica de la muestra bajo estudio y, en la segunda, correspondiente a la parte inferencial, se realizó un análisis factorial exploratorio (AFE) con las PAR que fueron medidas bajo la escala de Likert, de modo que se obtuvo el número de factores a trabajar y la medida de adecuación de Kaiser-Meyer-Olkin. También, aportó la prueba de esfericidad de Barlett, en la cual se evidenció que fue adecuado realizar el AFE como método estadístico para el cumplimiento del objetivo de la investigación.

Una vez obtenidos los resultados del AFE, se realizó un análisis de regresión logística (ARL). El concepto de regresión, tal como lo manifiestan Fiuza y Rodríguez (2000), hace referencia a la relación entre variables correlacionadas, por tanto, esta técnica permite explicar el comportamiento de una variable dependiente, en función de una o más variables independientes. Para este caso se tomaron como variables dependientes cada una de las dimensiones del compromiso organizacional, como variables independientes los indicadores PAR cuyas respuestas fueron dicotómicas, y los factores F1 y F2 obtenidos del AFE. Para el ARL, se utilizó el procedimiento de Backward:Wald, incorporado en el software utilizado. El análisis permitió evidenciar la incidencia de las PAR en el compromiso organizacional, de manera que se establece el logro del objetivo principal de la investigación. 


\section{Resultados}

$\mathrm{Al}$ aplicar el AFE se pudo constatar que los datos se ajustan de manera significativa para realizar este tipo de análisis. Como se observa en la tabla 6, la medida de adecuación de Kaiser-Meyer-Olkin es 0,869, lo cual implica que las magnitudes de los coeficientes de correlación parcial son pequeños, por tanto, es adecuado realizar el AFE; además, la prueba de esfericidad de Barlett es significativa, lo que indica que es procedente el análisis $(\mathrm{p}$-value $<0,05)$.

Tabla 6. KMO y prueba de Bartlett

\begin{tabular}{|c|c|c|}
\hline \multicolumn{2}{|c|}{ Medida de adecuación muestral de Kaiser-Meyer-Olkin. } & 0,869 \\
\hline \multirow{3}{*}{ Prueba de esfericidad de Bartlett } & Chi-cuadrado aproximado & 487,814 \\
\cline { 2 - 3 } & gl & 45 \\
\cline { 2 - 3 } & p-value & 0,000 \\
\hline
\end{tabular}

Fuente. Elaboración propia en IBM SPSS versión 23.

De esta manera, obtenidos los resultados y realizado el análisis, en la tabla 7 se aprecia que los dos primeros factores recogen el 60,812 \% de la variabilidad total de los datos, mientras que los otros ocho factores agrupan el 39,188 \%. A fin de conocer el número de factores que serían extraídos del AFE, se utilizó el criterio de Kaiser (Lloret, Ferreres y Hernández, 2014), el cual consiste en elegir aquellos factores del AFE que tengan autovalores o valores propios iniciales mayores a la unidad (1) (véase la Tabla 7).

Tabla 7. Varianza total explicada

\begin{tabular}{|c|c|c|c|c|c|c|}
\hline \multirow{2}{*}{ Componente } & \multicolumn{4}{|c|}{ Autovalores iniciales } & \multicolumn{2}{c|}{ Sumas de las saturaciones al cuadrado de la extracción } \\
\cline { 2 - 7 } & Total & $\begin{array}{c}\text { \% de la } \\
\text { varianza }\end{array}$ & \% acumulado & Total & $\begin{array}{c}\text { \% de la } \\
\text { varianza }\end{array}$ & \% acumulado \\
\hline 1 & 4,898 & 48,977 & 48,977 & 4,898 & 48,977 & 48,977 \\
\hline 2 & 1,183 & 11,834 & 60,812 & 1,183 & 11,834 & 60,812 \\
\hline 3 & 0,805 & 8,045 & 68,857 & & & \\
\hline 4 & 0,716 & 7,160 & 76,016 & & & \\
\hline 5 & 0,626 & 6,258 & 82,275 & & & \\
\hline 6 & 0,504 & 5,040 & 87,314 & & & \\
\hline 7 & 0,402 & 4,021 & 91,335 & & & \\
\hline 8 & 0,341 & 3,412 & 94,747 & & & \\
\hline 9 & 0,269 & 2,694 & 97,441 & & & \\
\hline 10 & 0,256 & 2,559 & 100,000 & & & \\
\hline
\end{tabular}

Fuente. Elaboración propia en IBM SPSS versión 23.

La matriz de componentes rotados en la tabla 8 , una vez utilizado el método de extracción de componentes principales, al igual que la rotación Varimax, agrupa las PAR en dos factores F1 y F2, tal como se muestra en la tabla 9. Posteriormente, a fin de dar respuesta a la correlación entre las variables de estudio, se realizó el ARL con cada una de las dimensiones del compromiso organizacional, lo que incluyó también las PAR con respuestas dicotómicas; se utilizó el procedimiento de 
Backward:Wald, el cual parte de un modelo con todas las variables que se hayan seleccionado y elimina de manera progresiva aquellas sin significancia estadística. Esto quiere decir que solo permanecerán las variables correlacionadas positivamente cuando los incrementos en los valores de una estén asociados estadísticamente a los valores de la otra.

Tabla 8. Matriz de componentes rotados

\begin{tabular}{|c|c|c|}
\hline \multirow{2}{*}{} & \multicolumn{2}{|c|}{ Componente } \\
\cline { 2 - 3 } & F1 & F2 \\
\hline PATE1 & $-0,052$ & 0,885 \\
\hline PAPR1 & 0,355 & 0,683 \\
\hline PARE1 & 0,699 & 0,169 \\
\hline PAPI1 & 0,616 & 0,394 \\
\hline PAED2 & 0,732 & 0,189 \\
\hline PAPV1 & 0,800 & 0,015 \\
\hline PACF1 & 0,826 & 0,166 \\
\hline PAEN1 & 0,722 & 0,074 \\
\hline PAMP1 & 0,797 & 0,081 \\
\hline PAEC1 & 0,723 & 0,194 \\
\hline
\end{tabular}

Fuente. Elaboración propia en IBM SPSS versión 23.

Tabla 9. Caracterización de las PAR respecto al análisis factorial exploratorio

\begin{tabular}{|c|c|c|c|}
\hline Abreviatura utilizada & Práctica de alto rendimiento evaluada & Forma de medición & Variable según AFE \\
\hline PADP1 & Desempeño basado en el pago & Dicotómica & N/A \\
\hline PADP2 & Desempeño basado en el pago & Dicotómica & N/A \\
\hline PATE1 & Trabajo en equipo & E. Likert & F2 \\
\hline PAPR1 & Participación & E. Likert & F1 \\
\hline PARE1 & Reducción de estatus & E. Likert & F1 \\
\hline PAPI1 & Promoción interna & E. Likert & F1 \\
\hline PAED1 & Sistemas formales de evaluación del \\
desempeno & Dicotómica & F1 \\
\hline PAED2 & Sistemas formales de evaluación del \\
desempeño & E. Likert & F1 \\
\hline PAPV1 & Progreso valorativo & E. Likert & N/A \\
\hline PACF1 & Programas de comunicación formal & E. Likert & F1 \\
\hline PAEN1 & Uso regular de encuestas de actitud de & Dicotómica & F1 \\
\hline PAMP1 & Méritos basados en la promoción & E. Likert & F1 \\
\hline PAEC1 & Estructura del cargo & E. Likert & Entrenamiento \\
\hline
\end{tabular}

Fuente. Elaboración propia. 
$\mathrm{Al}$ observar los factores que influyen en el hecho de que los empleados tengan o no un compromiso afectivo (véase la Tabla 10), se obtuvo que por cada unidad que se incremente el F1, la probabilidad de que los empleados manifiesten un compromiso afectivo se incrementa 3,27 veces; esta misma probabilidad se incrementa en 1,622 veces cuando F2 se incrementa en una unidad. Por otra parte, la probabilidad de que un empleado muestre un compromiso afectivo hacia la organización se incrementa en 2,074 veces entre los empleados cuando se implementa la variable PAED1, la cual hace referencia a la práctica denominada $\ll$ sistemas formales de evaluación del desempeño».

En otras palabras, se evidencia que las PAR inciden positivamente en el compromiso afectivo de los colaboradores en la caja de compensación familiar. Estos resultados son coherentes con la investigación realizada por $\mathrm{Hu}$ et al. (2019), quienes lograron comprobar cómo aquellas organizaciones que implementaban PAR permitían crear entornos de aprendizaje que contribuían a que los individuos mejoraran su trabajo y desarrollaran su autonomía $y$ afinidad, de manera que repercute positivamente en altos niveles de compromiso afectivo.

Tabla 10. Factores que influyen en el CA

\begin{tabular}{|c|c|c|c|}
\hline \multirow{2}{*}{ Variable } & \multicolumn{3}{|c|}{ Compromiso afectivo } \\
\cline { 2 - 4 } & $\boldsymbol{\beta}$ & Sig. $\mathbf{p}$-value & $\operatorname{Exp}(\boldsymbol{\beta})$ \\
\hline F1 & 1,187 & 0,000 & 3,278 \\
\hline F2 & 0,484 & 0,044 & 1,622 \\
\hline PAED1 & 0,715 & 0,009 & 2,074 \\
\hline
\end{tabular}

Fuente. Elaboración propia en IBM SPSS versión 23.

Ahora bien, al analizar los factores que influyen en el compromiso normativo, se aprecia que solo aquellas obtenidas del AFE fueron significativas; en este sentido, tal como se muestra en la tabla 11, se tiene que la probabilidad de tener un compromiso normativo con la empresa se incrementa 2,196 veces por cada unidad que se incremente F1, y 1,584 veces por cada unidad que se incremente F2. En este caso, no hubo PAR dicotómicas con significancia en el compromiso normativo.

A fin de contrastar este resultado es necesario considerar que este tipo de compromiso está relacionado con el concepto de reciprocidad, en el cual el individuo realiza esfuerzos para alcanzar los propósitos de la organización, en la medida en que la compañía demuestre, a través de la implementación de PAR, su interés en el desarrollo de sus habilidades (Rhee et al., 2018). Esto quiere decir que las PAR aplicadas por la caja de compensación las perciben por sus colaboradores como un mecanismo de retención que está generando un compromiso basado en la obligación de permanecer vinculado a la compañía (Allen y Meyer, 1996).

Tabla 11. Factores que influyen en el CN

\begin{tabular}{|c|c|c|c|}
\hline \multirow{2}{*}{ Variable } & \multicolumn{3}{|c|}{ Compromiso normativo } \\
\cline { 2 - 4 } & $\boldsymbol{\beta}$ & Sig. $\mathbf{p}$-value & $\operatorname{Exp}(\boldsymbol{\beta})$ \\
\hline F1 & 0,787 & 0,001 & 2,196 \\
\hline F2 & 0,460 & 0,037 & 1,584 \\
\hline
\end{tabular}

Fuente. Elaboración propia en IBM SPSS versión 23.

Finalmente, en el ARL realizado para el compromiso de continuidad se encontró que ninguna variable PAR estuvo relacionada, lo cual podría deberse a que los individuos vinculados a la caja de compensación pueden no estar percibiendo de parte del departamento de talento humano algún tipo de beneficio que se considere una pérdida en el momento que decidan dejar de pertenecer a la organización (Yahaya y Ebrahim, 2016). En otras palabras, las PAR implementadas por la caja de compensación no se perciben como una pérdida para los individuos a la hora de decidirse a encontrar un empleo diferente. 


\section{Conclusiones}

$\mathrm{L}$ as cajas de compensación en Colombia son instituciones sin ánimo de lucro con una función social, expresada en el desarrollo de planes que permiten mejorar la calidad de vida de la fuerza laboral y sus familias. Sin embargo, a fin de dar cumplimiento a su propósito es necesario contar con capital humano comprometido que lleve a cabo las actividades correspondientes a su misión. Por tanto, el desarrollo de este estudio contribuyó a dar respuesta a la pregunta de si existe o no incidencia de las PAR en el compromiso organizacional de los colaboradores en una caja de compensación en Colombia. Los resultados hallados permitieron evidenciar que existe una incidencia positiva de estas prácticas en, al menos, dos de las tres dimensiones abordadas del compromiso organizacional —afectivo y normativo-.

Estos resultados han sido consistentes con otros estudios (Fragoso et al., 2019; Tejada y Arias, 2005), lo cual podría explicarse por caracteristícas como, por ejemplo, el tamaño y la cultura de la organización, pues cuando se tienen pocos niveles de jerárquia o los entornos empresariales están dispuestos para el aprendizaje, los individuos se apropian de las PAR con mayor facilidad y presentan altos niveles de compromiso (Andersén y Andersén, 2019; Hu et al., 2019). En otras palabras, esto podría indicar que los colaboradores de la caja de compensación permanecen como miembros porque tienen un vínculo afectivo hacia la organización o porque sienten la obligación de permanecer debido a los beneficios recibidos.

Por otra parte, los resultados no permitieron determinar la relación entre las PAR y el compromiso de continuidad, lo cual es coherente con lo hallado en anteriores investigaciones (Grueso, 2007; Meyer y Smith, 2000). Explicado en palabras de Fabi et al. (2015), esto puede ser porque este tipo de compromiso difere en su naturaleza de los otros dos y, además, refiere a los costos percibidos de una eventual salida de la institución, aspecto que muchas veces no lo consideran relevante los colaboradores con miras a seguir realizando esfuerzos y continuar como miembros de una empresa (Yahaya y Ebrahim, 2016).

No obstante, los hallazgos de este estudio difieren de lo encontrado por Fragoso et al., (2019), quienes en su investigación realizada en la Armada de Portugal lograron comprobar que sí existía una relación positiva entre las PAR y el compromiso de continuidad, lo cual podría deberse a que los militares perciben que este tipo de prácticas pueden traerles algunos beneficios, razón por la que continuarán esforzándose por cumplir con sus tareas y responderán con actitudes positivas hacia esa institución.

Finalmente, se puede concluir que estos resultados dejan implicaciones importantes tanto para el ámbito académico como para la gestión empresarial. En primer lugar, se contribuye a la literatura de recursos humanos en un contexto latinoamericano y se aporta a la comprensión del compromiso como variable actitudinal que permite alcanzar un mejor desempeño a la organización. Además, los resultados permitieron contrastar lo realizado en otros estudios, en los que los autores sugieren utilizar el compromiso afectivo como el mejor predictor de compromiso, debido a que expresa el deseo del individuo a mantener una relación valiosa con la organización y promueva lealtad (Para-González et al., 2019).

En segundo lugar, para la caja de compensación familiar este trabajo significa que las prácticas implementadas han fortalecido un vínculo afectivo con sus colaboradores y se encuentran alineadas con el propósito misional, esto es, generar bienestar a los miembros de su comunidad. De igual forma, los resultados permiten a la dirección general 
continuar con su gestión y emprender acciones que no solo garanticen la continuidad de los hallazgos positivos, sino que impacten en su estrategia corporativa, la cual se verá retribuida en el servicio prestado a sus afiliados.

Por otra parte, se aporta al cambio de mentalidad de los directivos de las empresas, quienes han visto la implementación de las prácticas de recursos humanos desarticulada de la estrategia y con un fin netamente operativo (Pantoja-Kauffamann, 2019). Además, se recomienda tener en cuenta dos aspectos que son contribuir a la mejora del compromiso de las personas mediante la promulgación efectiva de las PAR y del comportamiento de liderazgo orientado a las relaciones (Gilbert, De Winne y Sels, 2011), y administrar adecuadamente los sistemas de PAR porque los colaboradores perciben diferentes significados, lo cual puede afectar el nivel de compromiso hacia la organización (Kloutsiniotis et al., 2021).

Como limitaciones de este estudio se presentan varios aspectos que vale la pena mencionar. Primero, los resultados obtenidos deben ser interpretados con cautela, debido a que el empírico fue realizado con una muestra extraída de una sola institución, razón por la que los hallazagos no podrían ser transferibles o generalizables en otros contextos similares. Segundo, la investigación se realizó bajo un enfoque transversal, de modo que se considera necesario realizar estudios longitudinales que permitan explicar mejor el comportamiento de estas variables en distintos momentos del tiempo $y$ en un contexto empresarial específico como es el de instituciones sin ánimo de lucro. Tercero, la literatura proveniente de estudios realizados en Colombia es escasa, motivo por el cual fue díficil realizar contrastaciones con aportes hechos en sectores empresariales y geográficos similares al seleccionado.
$\mathrm{Al}$ tomar en consideración las limitaciones, se recomienda para futuras investigaciones seguir analizando en empresas colombianas la efectividad de las PAR no solo en el compromiso organizacional, sino también en otras actitudes tales como intención de rotación, satisfacción laboral, sentido de pertenencia y cultura organizacional, entre otras. Así mismo, resulta necesario ampliar la investigación a otros sectores económicos con el ánimo de llevar a cabo comparativos y obtener conclusiones que permitan aportar a la teoría y al desarrollo de las organizaciones.

\section{Referencias}

Allen, N.; Meyer, J. (1996). Affective, continuance, and normative commitment to the organization: an examination of construct validity. Journal of Vocational Behavior, 49, 252-276. DOI: https://doi.org/10.1006/jvbe.1996.0043

Almutawa, Z.; Muenjohn, N.; Zhang, J. (2016). The effect of human resource management system on employees' commitment: the mediating role of the AMO model. The Journal of Developing Areas, 50(6), 17-29. DOI: https://doi. org/10.1353/jda.2016.0147

Andersén, J.; Andersén, A. (2019). Are high-performance work systems (HPWS) appreciated by everyone? The role of management position and gender on the relationship between HPWS and affective commitment. Employee Relations, 41(5), 1046-1064. DOI: https://doi.org/10.1108/ER-03-20180080

Andreassi, J.; Lawter, L.; Brockerhoff, M.; Rutigliano, P. (2014). Cultural impact of human resource practices on job satisfaction. a global study across 48 countries. Cross Cultural Management, 21(1), 55-77. DOI: https://doi.org/10.1108/ CCM-05-2012-0044

Angle, H.; Perry, J. (1981). An empirical assessment of organizational commitment and organizational effectiveness. Administrative Science Quarterly, 26, 1-14. DOI: https://doi. org $/ 10.2307 / 2392596$

Arthur, J. B. (1994). Effects of human resource systems on manucfacturing performance and turnover. Academy Management of Journal, 37(3), 670-687. DOI: https://doi. org/10.5465/256705

Barney, J. (1991). Firm resources and sustained competitive advantage. Journal of Managment, 17(1), 99-120. DOI: https://doi.org/10.1177\%2F014920639101700108 
Batt, R. (2002). Managing customer services: human resource pratices, quit rates and sales growth. Academy of Management Journal, 45(3), 587-597. DOI: https://doi. org/10.5465/3069383

Becker, B.; Huselid, M. (1998). High performance work systems and firm performance: a synthesis of research and managerial implications. Research in Personnel and Human Resource Management, 16, 53-101. Recuperado de https://bit. ly/2WZGF9z

Becker, H. (1960). Notes on the concept of commitment. American Journal of Sociology, 66(1), 32-40. DOI: https://doi. org $/ 10.1086 / 222820$

Bentein, K.; Vandenberghe, C.; Vandenberg, R.; Stinghamber, F. (2005). The role of change in the relation between commitment anda turnover: a latent growth modeling approach. Journal Applied of Psychology, 90(3), 468-482. DOI: https://doi.org/10.1037/0021-9010.90.3.468

Blau, P. (1964). Exchange and power in social ufe. Nueva York: Wiley.

Calderón-Hernánez, G.; Naranjo-Valencia, J.; Álvarez-Giraldo, C. (2010). Gestión humana en la empresa colombiana: sus características, retos y aporte. Cuadernos de Administración, 23(41), 13-36.

Chuang, C. H.; Chen, S.; Chuang, C. W. (2013). Human resource management practices and organizational social capital: the role of industrial characteristics. Journal of Business Research, 66(5), 678-687. DOI: https://doi.org/10.1016/j. jbusres.2012.04.002

Chuang, C. H.; Liao, H. (2010). Strategic human resource management in service context: taking care of business by taking care of employees and customers. Personnel Psychology, 63(1), 153-196. DOI: https://doi.org/10.1111/j.1744 6570.2009.01165.x

Cohen, A. (2008). Multiple commitments in the workplace. An integrative approach. Londres: Lawrence Erlbaum Associates.

Combs, J.; Liu, Y.; Hall, A.; Ketchen, D. (2006). How much do high-performance work practices matter? A meta-analysis of their effects on organizational performance. Personnel Psychology, 59(3), 501-528. DOI: https://doi.org/10.1111/ j.1744-6570.2006.00045.x

Datta, D.; Guthrie, J.; Wright, P. (2005). HRM and firm productivity: does industry matter? Academy of Management Journal, 48(1), 135-146. DOI: https://doi.org/10.5465/ amj.2005.15993158

Delaney, J.; Huselid, M. (1996). The impact of human resources management practices on perceptions of organizational performance. The Academy of Management Journal, 39(4), 949-969. DOI: https://doi.org/10.5465/256718
Delery, J.; Doty, H. (1996). Modes of theorizing in strategic human resource management: test of universalistic, contingency and configurational performance predictions. The Academy of Management Journal, 39(4), 802-835. DOI: https://doi.org/10.5465/256713

Fabi, B.; Lacoursière, R.; Raymond, L. (2015). Impact of highperformance work systems on job satisfaction, organizational commitment, and intention to quit in Canadian organizations. International Journal of Manpower, 36(5), 772-790. DOI: https://doi.org/10.1108/IJM-01-2014-0005

Ferrer, D. (2014). Prácticas de recursos humanos de alto rendimiento (Tesis de pregrado). Universidad de Almería. Almería, España. Recuperado de https://bit.ly/3lBZcmB

Fiuza, M.; Rodríguez , J. (2000). La regresión logística: una herramienta versátil. Nefrología, 20(6), 477-565. Recuperado de https://bit.ly/3ypCPEv

Fragoso, P.; Chambel, M.; Castanheira, F. (2019). The highperformance work system and commitment of the Portuguese Navy personnel. Military Psychology, 31(3), 251-266. DOI: https://doi.org/10.1080/08995605.2019.1598226

Frías-Navarro, D. (2020). Apuntes de consistencia interna de las puntuaciones de un instrumento de medida. Universidad de Valencia. Recuperado de https://bit.ly/3lr92rg

George, D.; Mallery, P. (2013). SPSS for Windows step by step: a simple guide and reference. 11.0 update (4a ed.). Boston: Allyn y Bacon.

Gilbert, C.; De Winne, S.; Sels, L. (2011). The influence of line managers and HR department on employees' affective commitment. The International Journal of Human Resource Management, 22(8), 1618-1637. DOI: https://doi.org/10.10 $80 / 09585192.2011 .565646$

Grueso, M. (2007). Impacto de las buenas prácticas de formación en el desarrollo del compromiso con la organización. Cuadernos de Administración, 23(38), 95-101. DOI: https:// doi.org/10.25100/cdea.v23i38.371

Guthrie, J. (2001). High-involvement work practices, turnover, and productivity: evidence form New Zeland. Academy Management of Journal, 44(1), 180-190. DOI: https://doi. org $/ 10.2307 / 3069345$

Haar, J.; O'Kane, C.; Daellenbach, U. (2021). High performance work systems and innovation in New Zealand SMEs: testing firm size and competitive environment effects. The International Journal of Human Resource Management. DOI: https://doi.org/10.1080/09585192.2021.1894213

Hu, B.; Hou, Z.; Chi Kuan Mak, M.; Lingxiao Xu, S.; Yang, X.; $\mathrm{Hu}, \mathrm{T}$.; Wen, Y. (2019). Work engagement, tenure, and external opportunities moderate perceived high-performance work systems and affective commitment. Social Behavior and Personality, 47(5), 1-16. DOI: https://doi.org/10.2224/ sbp.7353 
Huselid, M. (1995). The impact of human resource management on turnover, productivity and corporate financial performance. Academy Management of Journal, 38(3), 635-672. DOI: https://doi.org/10.5465/256741

Jaros, S.; Jermier, J.; Koehler, W.; Sincich, T. (1993). Effects of continuance, affective, and moral commitment on the withdrawal process: an evaluation of eight structural equation models. Academy Management of Journal, 36(5), 951-995. DOI: https://doi.org/10.5465/256642

Kehoe, R.; Wright, P. (2013). The impact of high- performance human resources practices on employees'attitudes and behaviors. Journal of Management, 39(2), 366-391. DOI: https://doi.org/10.1177\%2F0149206310365901

Khilji, S.; Wang, X. (2006). Intended and implemented HRM: the missing linchpin in strategic human resource management research. International Journal of Human Resource Management, 17(7), 1171-1189. DOI: https://doi. org/10.1080/09585190600756384

Klein, H.; Molloy, J.; Brinsfield, C. (2012). Reconceptualizing workplace commitment to redress a stretched construct: revisiting assumptions and removing confounds. Academy of Management Review, 37 (1), 130-151. DOI: https://doi. org/10.5465/amr.2010.0018

Kloutsiniotis, P.; Katou, A.; Mihail, D. (2021). Examining the «dark-side» of high performance work systems in the Greek manufacturing sector. Employee Relations: The International Journal, DOI: https://doi.org/10.1108/ER-04-2020-0170

Kloutsiniotis, P.; Katou, A.; Mihail, D. (2021). Examining the «dark-side» of high performance work systems in the Greek manufacturing sector. Employee Relations: The International Journal, 43(5),1104-1129. DOI: https://doi.org/10.1108/ ER-04-2020-0170

Lado, A.; Wilson, M. (1994). Human resources systems and sustained advantage: a competency- based perspective. The Academy of Management Review, 19(4), 699-727. DOI: https://doi.org/10.5465/amr.1994.9412190216

Lloret-Segura, S.; Ferreres-Trave, A.; Hernández-Baeza, A.; Tomás-Marco, I. (2014). El análisis factorial exploratorio de los ítems: una guía práctica, revisada y actualizada. Anales de Psicología, 30(3), 1151-1169. DOI: https://doi.org/10.6018/ analesps.30.3.199361

Macky, K.; Boxall, P. (2007). The relationship between highperformance work practices and employee attitudes: an investigation ofadditiveandinteractioneffects. TheInternational Journal of Human Resource Management, 18(4), 537-567. DOI: https://doi.org/10.1080/09585190601178745

Mahmood, A.; Akhtar, M.; Talat, U.; Shuai, C.; Hyatt, J. (2019). Specific HR practices and employee commitment: the mediating role of job satisfaction. Employee Relations, 41(3), 420-435. DOI: https://doi.org/10.1108/ER-03-2018-0074
Mayer, R.; Schoorman, D. (1992). Predicting participation and production outcomes through a two dimensional model of organizational commitment model. The Academy Management of Journal, 35(3), 671-684. DOI: https://doi. org/10.5465/256492

McDuffie, J. (1995). Human resource bundles and manufacturing performance: organizational logic and flexible production systems in the world auto industry. Industrial and Labor Relations Review, 48(2), 197-221. DOI: https://doi. org/10.1177\%2F001979399504800201

Meyer, J.; Allen, N. (1997). Commitment in the workplace. Theory, Research and Application. Thousand Oaks: Sage.

Meyer, J.; Allen, N.; Smith, K. (1993). Commitment to organizations and occupations. Extension and test of a threecomponent conceptualization. Journal of Applied Psychology, 78(4), 538-551. DOI: https://doi.org/10.1037/00219010.78.4.538

Meyer, J.; Stanley, D.; Herscovitch, L.; Topolnytsky, L. (2002). Affective, continuance, and normative commitment to the organization: a meta-analysis of antecedents, correlates and consequences. Journal of Vocational Behavior, 61(1), 20-52. DOI: https://doi.org/10.1006/jvbe.2001.1842

Meyer, J.; Stanley, D.; Jackson, T.; McInnis, K.; Maltin, E. R.; Sheppard, L. (2012). Affective, normative, and continuance commitment levels across cultures: a meta-analysis. Journal of Vocational Behavior, 80(2), 225-245. DOI: https://doi. org/10.1016/j.jvb.2011.09.005

Meyer, P.; Smith, A. (2000). HRM practices and organizational commitment: test of a mediation model. Canadian Journal of Administrative Sciences, 17(4), 319-331. DOI: https://doi. org/10.1111/j.1936-4490.2000.tb00231.x

Miao, R.; Bozionelos, N.; Zhou, W.; Newman, A. (2021). Highperformance work systems and key employee attitudes: the roles of psychological capital and an interactional justice climate. The International Journal of Human Resource Management, 32(2), 443-477. DOI: https://doi.org/10.1080 /09585192.2019.1710722

Mierlo, J.; Bondarouk, T.; Sanders, K. (2018). The dynamic nature of HRM implementation: a structuration perspective. The International Journal of Human Resource Management, 29(22), 3026-3045. DOI: https://doi.org/10.1080/095851 92.2018 .1443957

Mowday, R. (1998). Reflections on the study and relevance of organizational commitment. Human Resource Management Review, 8(4), 387-401. DOI: https://doi.org/10.1016/ S1053-4822(99)00006-6

Nishii, L.; Lepak, D.; Schneider, B. (2008). Employee attributions of the «why» of HR practices: their effects on employee attitudes and behaviors, and customer satisfaction. Personnel Psychology, 61(3), 503-545. DOI: https://doi.org/10.1111/ j.1744-6570.2008.00121.x 
O’Reilly, C.; Chatman, J. (1986). Organizational commitment and psychological attachment: the effects of compliance, identification, and internalization on prosocial behavior. Journal of Applied Psychology, 71(3), 492-499. DOI: https:// doi.org/10.1037/0021-9010.71.3.492

Pantoja-Kauffmann, G. (2019). Gestión del talento humano en micro, pequeñas y medianas empresas de servicios en Colombia: aproximaciones a un modelo de diagnóstico organizacional. Revista Escuela de Administración de Negocios, (87), 13-26. DOI: https://doi.org/10.21158/01208160. n87.2019.2441

Para-González, L.; Jiménez-Jiménez, D.; Martínez-Lorente, Á. R. (2019). Do SHRM and HPWS shape employees' affective commitment and empowerment? Evidence-based HRM, 7(3), 300-324. DOI: https://doi.org/10.1108/ EBHRM-01-2019-0004

Pattnaik, S.; Sahoo, R. (2019). High-performance work practices, affective commitment of employees and organizational performance: a multi-level modelling using 2-1-2 mediation analysis. Global Business Review, 22(6), 1594-1609. DOI: https://doi.org/10.1177/0972150919859106

Pfeffer, J. (1998). Seven practices of successful organizations. California Management Review, 40(2), 96-124. DOI: https:// doi.org/10.2307/41165935

Porter, L.; Steers, R.; Mowday, R.; Boulian, P. V. (1974). Organizational commitment, job satisfaction and turnover among psychiatric technicians. Journal of Applied Psychology, 59(5), 603-609. DOI: https://doi.org/10.1037/h0037335

Rhee, S-Y.; Oh, H. Y.; Yu, G. J. (2018). High-performance work systems and firm capabilities in Korea: a fit perspective with organizational culture. Asia Pacific Journal of Human Resources, 56(3), 317-340. DOI: https://doi.org/10.1111/17447941.12134

Rodríguez-Arévalo, V. (2012). La naturaleza jurídica de las cajas de compensación en Colombia: una visión frente a su control. Justicia Juris, 8(1), 9-21. DOI: https://doi.org/10.15665/ rj.v8i1.247

Salancik, G. (1977). Commitments it too easy! Organizational Dynamics, 6(1), 62-80. DOI: https://doi.org/10.1016/00902616(77)90035-3

Singh, A.; Gupta, B. (2015). Job involvement, organizational commitment, professional commitment, and team commitment: a study of generational diversity. Benchmarking: An International Journal, 22(6), 1192-1211. DOI: https://doi. org/10.1108/BIJ-01-2014-0007

Sun, Y.; Mamman, A. (2021). Adoption of high-performance work systems in small and medium-sized enterprises. Asia Pacific Journal Human Resource. DOI: https://doi. org/10.1111/1744-7941.12277
Takeuchi, R.; Way, S.; Wei Tian, A. (2018). Cross-lebes effects of support climate: main and moderating roles. Human Resource Management, 57(5), 1205-1218. DOI: https://doi. org $/ 10.1002 / \mathrm{hrm} .21891$

Tejada, T.; Arias, G. (2005). Prácticas organizacionales y el compromiso de los trabajadores hacia la organización. Enseñanza e Investigación en Psicología, 10(2), 295-309.

Way, S. (2002). High performance work systems and intermediate indicators of firm performance within the US small business sector. Journal of Management, 28(6), 765-785. DOI: https:// doi.org/10.1016/S0149-2063(02)00191-5

Whitener, E. (2001). Do high commitment human resource practices affect employee commitment? Across-level analysis using hierarchical linear modeling. Journal of Management, 27(5), 515-535. DOI: https://doi. org/10.1177\%2F014920630102700502

Wong, I.; Gao, J. (2014). Exploring the direct and indirect effects of CRS on organizational commitment. The mediating role of corporate culture. International Journal of Contemporary Hospitality Management, 26(4), 500-525. DOI: https://doi. org/10.1108/IJCHM-05-2013-0225

Wright, P.; Gardner, T.; Moynihan, L. (2003). The impact of HR practices on the performance of business units. Human Resource Management Journal, 13(3), 21-36. DOI: https://doi. org/10.1111/j.1748-8583.2003.tb00096.x

Yahaya, R.; Ebrahim, F. (2016) Leadership styles and organizational commitment: literature review. Journal of Management Development, 35(2), 190-216. DOI: https://doi. org/10.1108/JMD-01-2015-0004 